\title{
A CHARACTERIZATION OF THE CLOSED 2-CELL*
}

\author{
BY \\ HASSLER WHITNEY†
}

1. Introduction. A number of characterizations have been given of the simple closed surface. $\ddagger$ The proofs involve considerable point set difficulties. We give here a characterization of the closed 2-cell, that is, a point set homeomorphic with a circle and its interior. The fundamental theorem is partly of a combinatorial and partly of a continuity nature. It reads

THEOREM I. Let $R$ be a continuous curve $\S$ containing the simple closed curve $J$, such that

(1) $J$ is irreducibly homologous to zero in $R$, and

(2) If $\gamma$ is an arc with just its two end points $a$ and $b$ on $J$, then $R-\gamma$ is not connected.

Let $R^{\prime}$ and $J^{\prime}$ be defined similarly. Then $R$ and $R^{\prime}$ are homeomorphic, with $J$ corresponding with $J^{\prime}$.

That $R$ is a closed 2-cell then follows immediately from the following theorem. We note that $J$ corresponds with the circle, that is, $J$ is the boundary of $R$.

THEOREM II. If $I$ is a circle in the plane and $S$ is I with its interior, then $S$ and I satisfy the conditions prescribed for $R$ and $J$ in the above theorem.

The exact meaning of Condition (1) of Theorem I is given in $\$ 4$; a stronger condition is the following: For every $\epsilon>0$ and any two points $a$ and $b$ on $J$, there is a set of points $a_{i j}$ in $R, 1 \leqq i \leqq m, 1 \leqq j \leqq n$, such that all points $a_{1 j}$ coincide with $a$, all points $a_{m j}$ coincide with $b$, all points $a_{i 1}$ lie on one arc $a b$ of $J$, all points $a_{i n}$ lie on the other $\operatorname{arc} a b$ of $J$, and $\|$

$$
\rho\left(a_{i j}, a_{i+1, j}\right)<\epsilon, \quad \rho\left(a_{i j}, a_{i, j+1}\right)<\epsilon ;
$$

moreover, this does not hold in any proper subset of $R$ containing $J$.

* Presented to the Society, October 31, 1931; received by the editors April 13, 1932.

$\dagger$ National Research Fellow.

$\ddagger$ That is, a point set homeomorphic with the surface of a sphere. See L. Zippin, American Journal of Mathematics, vol. 52 (1931), pp. 331-350; these Transactions, vol. 31 (1929), pp. 744-770; C. Kuratowski, Fundamenta Mathematicae, vol. 13 (1929), pp. 307-318; also references in these papers.

$\S$ See Lemma A.

$\| \rho(p, q)=$ distance from $p$ to $q$, or in general, distance between two point sets; $\delta(S)=$ diameter of $S ; V_{\epsilon}(S)=$ those points $p$ for which $\rho(p, S)<\epsilon ; W_{\epsilon}(S)=$ those points $p$ for which $\rho(p, S) \leqq \epsilon$. 
Notations and preliminary theorems are given in $\$ \S 2,3$ and 4 ; an outline of the proof of Theorem I will be found in $\$ 5$. The Jordan and related theorems follow of course from the above theorems.

2. Point set background. Elementary properties of point sets we shall need may be found in Hausdorff, Mengenlehre, chapter VI. A continuous curve is a metric space which can be expressed as the continuous image of a closed line segment. An arc is the topological image of a closed line segment; a simple closed curve, the topological image of a circle.

Two fundamental lemmas are the following:

Lemma A.* $A$ compact, connected and locally connected metric space is a continuous curve, and conversely.

LEмма B. $\dagger A$ continuous curve is arcwise connected.

That is, any two points $p$ and $q$ in the set are end points of an arc $p q$ in the set. Using the definition of a continuous curve, it is easily seen that two continuous curves which have common points form a continuous curve.

From these lemmas we deduce the following known theorems.

Lemma C. Any continuum $C$ of diameter $<\epsilon$ in a continuous curve $R$ is contained in a continuous curve $C^{\prime}$ in $R$ of diameter $<\epsilon$.

Say $\delta(C)=\epsilon-\epsilon^{\prime} . R$ being the continuous image of a closed line segment, we can divide this segment into segments so small that the diameter of the image of each is $\left\langle\epsilon^{\prime}\right.$. We let $C^{\prime}$ be the union of all of these images which have points in common with $C$.

LEMma D. A continuous curve $R$ is locally arcwise connected.

That is, given a point $p$ and an $\epsilon>0$, there is a $\delta>0$ such that if $q \subset V_{\delta}(p)$, then there is an arc $p q$ in $R$ of diameter $<\epsilon$. As $R$ is locally connected, we can take $\delta$ so that if $q \subset V_{\delta}(p)$, there is a continuum $C$ in $R$ of diameter $<\epsilon$ containing $p$ and $q$. The continuum $C$ is contained in a continuous curve $C^{\prime}$ of diameter $<\epsilon$, and $C^{\prime}$ is arcwise connected; hence there is an arc $p q \subset C^{\prime} \subset R$, and $\delta(p q)<\epsilon$.

$R$ is of course uniformly locally arcwise connected, by the Borel Theorem.

LEMMA E. A connected open subset $R^{\prime}$ of a continuous curve $R$ is arcwise connected.

* See G. T. Whyburn, Concerning continuous images of the interval, American Journal of Mathematics, vol. 53 (1931), pp. 670-674.

† See references in R. L. Moore, Report on continuous curves, Bulletin of the American Mathematical Society, vol. 29 (1923), p. 293, footnote $(\dagger)$. 
If there are two points $p$ and $q$ in $R^{\prime}$ which are joined by no arc in $R^{\prime}$, let $A$ contain $p$ and all points of $R^{\prime}$ joined to $p$ by an arc in $R^{\prime}$, and put $B=R^{\prime}-A$; then there is no arc joining a point of $A$ to a point of $B$ in $R^{\prime}$. As $R^{\prime}$ is connected, there is a point $p^{\prime}$ in one of these sets, say $B$, which is a limit point of points of the other set, $A$. As $R^{\prime}$ is open in $R, \rho\left(p^{\prime}, R-R^{\prime}\right)=$ $\epsilon>0$. We can take $q^{\prime}$ in $A$ so close to $p^{\prime}$ that there is an $\operatorname{arc} p^{\prime} q^{\prime}$ in $R$ of diameter $<\epsilon$. But then $p^{\prime} q^{\prime} \subset R^{\prime}$, a contradiction.

Suppose $R$ is connected, and $p \subset R$ is such a point that $R-p$ is not connected. Then $p$ is called a cut point of $R$.

Leмma F. Let $R$ be a continuous curve without a cut point. Then for every $\epsilon>0$ there is a $\delta>0$ such that if $\rho(q, p) \geqq \epsilon$ and $\rho\left(q^{\prime}, p\right) \geqq \epsilon$, then there is an arc $q q^{\prime}$ with no points in $V_{\delta}(p)$.

Suppose the contrary. Then there are three sequences of points $\left\{p_{n}\right\}$, $\left\{q_{n}\right\},\left\{q_{n}^{\prime}\right\}$, approaching points $p, q, q^{\prime}$, respectively, with $\rho\left(q_{n}, p_{n}\right) \geqq \epsilon$, $\rho\left(q_{n}^{\prime}, p_{n}\right) \geqq \epsilon$, and such that for each $n$, any arc $q_{n} q_{n}^{\prime}$ must contain points in $V_{\delta_{n}}\left(p_{n}\right)$, where $\lim _{n \rightarrow \infty} \delta_{n}=0$. By Lemma D it is seen that for any $n$ greater than some $N$ there are $\operatorname{arcs} q_{n} q, q_{n}^{\prime} q^{\prime}$, with no points in $V_{\epsilon / 2}(p)$. It follows that any arc $q q^{\prime}$ must pass through $p$, contradicting Lemma $\mathrm{E}$ (as $p$ is not a cut point).

3. Combinatorial background.* A $k$-simplex, or abstract $k$-simplex, is a set of $k$ elements (say points) $a_{1} a_{2} \cdots a_{k}$. The order in which we write the points is immaterial. For $k=0,1$ and 2 we use also the terms vertex, segment and triangle respectively. A $k$-chain is a set of $k$-simplexes, and is written as the sum of these simplexes. The sum $(\bmod 2)$ of several $k$-chains is the $k$-chain containing those simplexes which occur in an odd number of the $k$-chains.

The boundary $K$ of a $k$-simplex $L, k>0$, is the sum of all $(k-1)$-simplexes formed by dropping out one of the vertices of the simplex. We write $L \rightarrow K$. A 0 -simplex has no boundary. Thus

$$
a \rightarrow 0, a b \rightarrow a+b, a b c \rightarrow a b+a c+b c .
$$

The boundary of a $k$-chain is the sum $(\bmod 2)$ of the boundaries of the simplexes of the chain. Thus

$$
a b+b c+c d \rightarrow a+d, a b c+b c d \rightarrow a b+a c+b d+c d .
$$

Evidently the boundary of a sum of several $k$-chains is the sum of the boundaries of the chains. If a $k$-chain has no boundary, it is called a $k$-cycle. (Any 0-chain is a 0 -cycle.) The boundary of a $k$-chain $(k>0)$ is $a(k-1)$-cycle. This is evi-

\footnotetext{
* Compare L. Vietoris, Über den höheren Zusammenhang kompakter Räume, Mathematische Annalen, vol. 97 (1927), pp. 454-472.
} 
dent if the $k$-chain is a $k$-simplex. The general case then follows from the last theorem.

Lemma G. If $K \rightarrow a+b$ is a 1-chain, then there is a chain of segments aa $a_{1}$, $a_{1} a_{2}, \cdots, a_{n} b$ in $K$.

For otherwise we could divide the segments of $K$ into two groups $K_{1} \supset a$ and $K_{2} \supset b$, no two simplexes from different groups having a common vertex. But then $K_{1} \rightarrow a, K_{2} \rightarrow b$, which cannot be, as the boundary of any 1-chain contains an even number of vertices.

A 1 -circuit is a 1-cycle of the form $a_{1} a_{2}, a_{2} a_{3}, \cdots, a_{n-1} a_{n}, a_{n} a_{1}$, the vertices being distinct except as shown.

Lemma H. Any 1-cycle $K$ is a sum of 1-circuits.

If $a_{1} a_{n}$ is a segment of $K$, then $K+a_{1} a_{n} \rightarrow a_{1}+a_{n}$, as $K \rightarrow 0$ and $a_{1} a_{n} \rightarrow a_{1}+a_{n}$. We can thus find a set of distinct segments and vertices $a_{1} a_{2}, \cdots, a_{n-1} a_{n}$ in $K+a_{1} a_{n}$ not containing $a_{1} a_{n}$. This with $a_{1} a_{n}$ is a 1 -circuit $K_{1}$. As $K_{1} \rightarrow 0$, $K+K_{1}$ is a 1-cycle containing no segments of $K_{1}$, and it contains a 1-circuit $K_{2}$. Continuing, we find $K=K_{1}+K_{2}+\cdots+K_{m}$.

4. A $k$-chain $K$ is said to lie in a point set $R$ if each vertex of $K$ is in $R$. Any vertex now has both a name and a position. Two vertices are distinct if their names are distinct, irrespective of whether they coincide in position or not. $\epsilon$ being a positive number, a $k$-simplex $K \subset R$ is called an $(\epsilon, k)$-simplex in $R$ if $\delta(K)<\epsilon$, i.e. if any two vertices of $K$ are within $\epsilon$ of each other. A $k$ chain is an $(\epsilon, k)$-chain if each of its simplexes is an $(\epsilon, k)$-simplex. A $k$-cycle $K$ in $S$ is said to be $\epsilon$-homologous to zero $(K \epsilon \sim 0)$ in $R$ if there is an $(\epsilon, k+1)$ chain $L$ in $R$ of which $K$ is the boundary. If $K_{1} \epsilon \sim 0$ and $K_{2} \epsilon \sim 0$, then $K_{1}+K_{2}$ $\epsilon \sim 0$. We write also $K_{1} \epsilon \sim K_{2}$ for $K_{1}+K_{2} \epsilon \sim 0$. If $K_{1} \epsilon \sim K_{2}$ and $K_{2} \epsilon \sim K_{3}$, then $K_{1} \in \sim K_{3}$.

Suppose the closed set $R$ contains the simple closed curve $J$. If for every $\epsilon>0$ there is a $\delta>0$ such that any $(\delta, 1)$-cycle on $J$ is $\epsilon \sim 0$ in $R$, then we say that $J \sim 0$ in $R$. If $J$ is $\sim 0$ in $R$ but is not $\sim 0$ in any proper closed subset of $R$ containing $J$, then we say that $J$ is irreducibly $\sim 0$ in $R$.

LеммA I. Given a simple closed curve $J$, let us divide it into the arcs* $\overline{a_{1} a_{2}}$, $\overline{a_{2} a_{3}}, \cdots, \overline{a_{n-1} a_{n}}, \overline{a_{n} a_{1}}$, each of diameter $<\epsilon / 2$. Let $\delta$ be smaller than the distance between any two of these arcs which have no common points. Then if $K^{\prime}=a_{1} a_{2}+a_{2} a_{3}+\cdots+a_{n} a_{1}$ and $K$ is any $(\delta, 1)$-cycle on $J, K$ is either $\epsilon \sim 0$ or $\epsilon \sim K^{\prime}$ on $J$.

By Lemma $\mathrm{H}, K$ is a sum of 1 -circuits $K_{1}, \cdots, K_{m}$. If we show that each

* Here, $\overline{a_{1} a_{2}}$ denotes an arc, and $a_{1} a_{2}$, a segment. 
$K_{i}$ is $\epsilon \sim \alpha_{i} K^{\prime}, \alpha_{i}=0$ or 1 , it will follow that $K=\sum K_{i} \epsilon \sim \sum \alpha_{i} K^{\prime}=0$ or $K^{\prime}$ (depending on whether $\sum \alpha_{i}$ is even or odd), and the lemma will be proved.

Consider any $K_{i}=b_{1} b_{2}+b_{2} b_{3}+\cdots+b_{s} b_{1}$, say. If a vertex $b_{j}$ of $K_{i}$ does not lie on any point $a_{k}$, say $b_{j} \subset \overline{a_{k} a_{k+1}}$; add to $K_{i}$ the boundary of the $\epsilon$ triangles $b_{j-1} b_{j} a_{k}^{\prime}+b_{j} b_{j+1} a_{k}^{\prime}$, where $a_{k}{ }^{\prime}$ is a new vertex lying on $a_{k}$. The result is an $(\epsilon, 1)$-circuit $K_{i}^{(1)} \epsilon \sim K_{i}$, the vertex $b_{j}$ having been replaced by the vertex $a_{k}{ }^{\prime}$. Repeat the process till we have an $(\epsilon, 1)$-circuit $K^{\prime \prime}=c_{1} c_{2}+c_{2} c_{3}+\cdots$ $+c_{s} c_{1} \in \sim K_{i}$.

Now any two consecutive vertices $c_{j}, c_{j+1}$ lie on the same or consecutive vertices of $K^{\prime}$. Suppose $c_{j}$ is on $a_{k}$ and $c_{j+2}$ is on $a_{k+p}, p \neq 2$ or -2 . Then add the boundary of $c_{j} c_{j+1} c_{j+2}$, replacing the segments $c_{j} c_{j+1}+c_{j+1} c_{j+2}$ by the single segment $c_{j} c_{j+2}$. Continue till we arrive at a (possibly void) $(\epsilon, 1)$-circuit $K^{*}$ $=d_{1} d_{2}+\cdots+d_{r} d_{1} \epsilon \sim K_{i}$. If $d_{1}$ lies on $a_{k}$, then $d_{j+1}$ lies on $a_{k \pm i}$, where we put $n+p=p$, etc.

If $K^{*}$ contains no segments, $K_{i} \epsilon \sim 0$. Otherwise, following the vertices $d_{1}, d_{2}, \cdots, d_{r}, d_{1}$ of $K^{*}$, we have gone around $J p$ times say. Add to $K^{*}$ the boundaries of all the $2 r \epsilon$-triangles of the following sort. If $d_{j}$ lies on $a_{k}$, and $d_{j+1}$ on $a_{k \pm 1}$, two of the triangles are $d_{j} d_{j+1} a_{k}$ and $d_{j+1} a_{k} a_{k \pm 1}$. The result is an $(\epsilon, 1)$-cycle $p K^{\prime}=0$ or $K^{\prime}$. Thus $K_{i} \epsilon \sim 0$ or $K^{\prime}$, and the proof is complete.

An immediate consequence of this lemma is

Lemma J. Let the simple closed curve $J$ lie in the closed set $R$. If for every $\epsilon>0$ there is a 1-cycle $K^{\prime}$ in $J$ as above described which is $\epsilon \sim 0$ in $R$, then $J \sim 0$ in $R$.

LEMmA $\mathrm{K}$. If $\gamma$ is an arc, then for every $\epsilon>0$ there is a $\delta>0$ such that any $(\delta, 1)$-cycle on $\gamma$ is $\epsilon \sim 0$ on $\gamma$.

The proof below holds in fact if $\gamma$ is a closed $k$-cell, any $k$. It is sufficient to prove it for the case that $\gamma$ is a closed line segment, in which case we can take $\delta=\epsilon / 2$. $\dagger$

Let $K$ be a $(\delta, 1)$-cycle on $\gamma$, let $a_{0} b_{0}$ be a segment of $K$, and say $\delta(\gamma)=\alpha$. Choose a fixed point $p$ in $\gamma$, and an integer $n>\alpha / \delta$. Let the vertices $a_{1}$, $a_{2}, \cdots, a_{n-1}$ divide the segment $a_{0} p$ into $n$ equal parts, and similarly for the vertices $b_{1}, b_{2}, \cdots, b_{n-1}$. Add to $K$ the boundaries of all triangles of the form $a_{i} a_{i+1} b_{i}, a_{i+1} b_{i} b_{i+1}, a_{n-1} b_{n-1} p$, and of all similar triangles corresponding to the other segments of $K$. The result is 0 . As all the triangles employed are $\epsilon$ triangles, $K \epsilon \sim 0$ in $\gamma$.

5. Outline of the proof of Theorem I. The proof runs as follows.

$\dagger$ The essential point in the proof below is that $\gamma$ is convex: any two points of $\gamma$ are end points of a line segment in $\gamma$. The proof is then easily extended to the case of any set homeomorphic with $\gamma$. 
(a) In $\S 6$ we show how an arc $\gamma$ can be drawn in $R$ crossing $J, \dagger$ avoiding two given closed sets. $R-\gamma$ is not connected.

(b) In $\$ 7$ we prove some lemmas. These show ( $\$ 8)$ that $R-\gamma$ contains exactly two components $A^{\prime}$ and $B^{\prime}$. If $A=A^{\prime}+\gamma$, then $A$ and its boundary curve $J_{A}$ (which is $\gamma$ plus a part of $J$ ) satisfy condition (1) of the theorem; similarly for $B=B^{\prime}+\gamma$ and $J_{B}$. Further, $A$ and $B$ are continuous curves.

(c) In $\$ 9$ it is shown that any arc in $A$ (or $B$ ) crossing $J_{A}\left(J_{B}\right)$ divides $A(B)$. Thus $A$ and $J_{A}\left(B\right.$ and $\left.J_{B}\right)$ satisfy all the conditions of the theorem. Hence we can cut up each set just as we cut up $R$, and can continue indefinitely.

(d) The object of $\S 10$ is to prove that $R$ may be cut into pieces of arbitrarily small diameter.

(e) The homeomorphism between $R$ and $R^{\prime}$ is now easily established. We cut $R$ up indefinitely, and cut $R^{\prime}$ in a corresponding fashion. Any point $p$ of $R$ lies in a descending sequence of pieces; the corresponding sequence in $R^{\prime}$ determines a point $p^{\prime}$, which we let correspond to $p$.

We turn now to the detailed proof.

6 . An arc crossing $J$. We prove here

Lemma L. $\ddagger$ Let the simple closed curve $J$ be $\sim 0$ in the continuous curve $R$. Let $c$ and $d$ be two points of $J$, dividing $J$ into the two arcs $\eta_{1}$ and $\eta_{2}$. If $C$ and $D$ are two closed sets in $R$ containing $c$ and $d$ respectively, and $C \cdot D=0$, then there is an arc $\gamma$ in $R$ joining $\eta_{1}$ to $\eta_{2}$ which has no points in $C$ or in $D$.

Say $\rho(C, D)=3 \epsilon$, and put $C^{\prime}=W_{\epsilon}(C), D^{\prime}=W_{\epsilon}(D)$; then $\rho\left(C^{\prime}, D^{\prime}\right)=\epsilon$. Take $\sigma$ so small that any two points in $R$ within $\sigma$ of each other are joined by an arc of diameter $<\epsilon($ Lemma D). Take $\delta$ so small that any $(\delta, 1)$-cycle on $J$ is $\sigma \sim 0$ in $R$. Construct the $(\delta, 1)$-cycle $K=c c_{1}+c_{1} c_{2}+\cdots+c_{m} d+d d_{1}$ $+d_{1} d_{2}+\cdots+d_{n} c, c_{i} \subset \eta_{1}, d_{i} \subset \eta_{2}$. There is a $(\sigma, 2)$-chain

$$
L=L_{C}+L_{D} \rightarrow K
$$

in $R$, where we let $L_{C}$ contain all those triangles of $L$ with vertices in $C^{\prime}$, and let $L_{D}$ be the rest of $L$.

Say

$$
L_{C} \rightarrow K_{C}=K_{C}^{\prime}+K^{*},
$$

where we let $K_{C}^{\prime}$ contain all those segments of $K_{C}$ which are also in $K$. As $L_{C} \subset V_{\sigma}\left(C^{\prime}\right), K^{*} \cdot D^{\prime}=0$. Define $K_{D}{ }^{\prime}$ by the relation

$\dagger$ That is, $\gamma$ lies in $R$, and has only its end points on $J$.

$\ddagger$ Compare P. Urysohn, Über Räume mit verschwindender erster Brouwerscher Zahl, Proceedings. Amsterdam Akademie van Wetenschappen, vol. 31 (1928), pp. 808-810. 


$$
L_{D} \rightarrow K_{D}=K_{D}^{\prime}+K^{*} \text {. }
$$

Adding these relations gives $L$ on the left, and hence $K$ on the right:

$$
K=K_{C}^{\prime}+K_{D}^{\prime} \text {. }
$$

As all the segments of $K_{c}^{\prime}$ are in $K, K_{D}^{\prime}$ must contain just those segments of $K$ not in $K_{C^{\prime}}$; in particular, it contains no segments of $K^{*}$. Hence all the segments of $K^{*}$ are present in $K_{D}{ }^{\prime}+K^{*}$, the boundary of $L_{D}$ (i.e. none have canceled out with segments of $K_{D}{ }^{\prime}$ ). Hence, as $L_{D} \cdot C^{\prime}=0$,

$$
K^{*} \cdot C^{\prime}=K^{*} \cdot D^{\prime}=0 \text {. }
$$

As $K_{C}$ is the boundary of $L_{C}$, it is a 1 -cycle; hence

$$
K_{C}+c c_{1}=K_{C}^{\prime}+K^{*}+c c_{1} \rightarrow c+c_{1} .
$$

By Lemma G, $K_{C}+c c_{1}$ contains a chain of segments joining $c_{1}$ to $c$. Following this chain, let $p_{s}$ be the first vertex in $\eta_{2}$, and $p_{0}$, the last vertex before $p_{s}$ in $\eta_{1}$, and say $p_{0} p_{1}, p_{1} p_{2}, \cdots, p_{s-1} p_{s}$ are the segments in between. We shall show that these segments are in $K^{*}$. If $s>1$ this is obvious, as then $p_{1}, \ldots$ $p_{s-1}$ exist and are not on $J$. Suppose $s=1$ and $p_{0} p_{1}$ is not in $K^{*}$; then it is in $K_{c}^{\prime}+c c_{1}$. It could only be the segment $c c_{1}$. But $c c_{1}$ lies in $K$ and not in $K_{D}$, hence it is in $K_{c}$; it is not in $K^{*}$, hence it is in $K_{C}+K^{*}=K_{C^{\prime}}$, and therefore not in $K_{C}^{\prime}+c c_{1}$. This proves the statement.

Now let $\overline{p_{i} p_{i+1}}$ be an arc of diameter $<\epsilon$ in $R, i=0, \cdots, s-1$. These arcs form a continuous curve, from which we can pick out an arc $\gamma$ (Lemma B) joining $\eta_{1}$ to $\eta_{2}$; we can take $\gamma$ so only its end points are on $J$. As $p_{i} p_{i+1} \subset K^{*}$ and $\delta\left(\overline{p_{i} p_{i+1}}\right)<\epsilon, \gamma$ has no points in $C$ or in $D$, and the lemma is proved.

7. We prove three lemmas.

Lemma M. If $J \subset C, J \sim 0$ in $C+D$, and $C \cdot D=\operatorname{an}$ arc $\gamma$, then $J \sim 0$ in $C$.

Given an $\epsilon>0$, choose first $\epsilon_{1}$ so small that any $\left(3 \epsilon_{1}, 1\right)$-cycle on $\gamma$ is $\epsilon \sim 0$ in $\gamma$ (Lemma K). Take next $\epsilon_{2}<\epsilon_{1}$ so that if $p \subset D$ and $\rho(p, C)<\epsilon_{2}$, then $\rho(p, \gamma)<\epsilon_{1}$. (If $D_{1}=D-D \cdot V_{\epsilon_{1}}(\gamma)$, take $\epsilon_{2}<\rho\left(D_{1}, C\right)$.) Take finally $\delta<\epsilon_{2}$ so that any $(\delta, 1)$-cycle $K$ on $J$ is $\epsilon_{2} \sim 0$ in $C+D$; we shall show that $K \epsilon \sim 0$ in $C$.

Let $L \rightarrow K$ be an $\left(\epsilon_{2}, 2\right)$-chain in $C+D$. Take any vertex $p$ of $L$ in $D \cdot V \epsilon_{\epsilon_{2}}(C)$ $-\gamma$, and replace it by a vertex $p^{\prime} \subset \gamma$, where $\rho\left(p, p^{\prime}\right)<\epsilon_{1} . L$ is thus replaced by a $\left(3 \epsilon_{1}, 2\right)$-chain $L^{\prime}$, in which each triangle lies wholly in either $C$ or $D$. Moreover, $L^{\prime} \rightarrow K$, as no vertices of $K$ have been moved.

Put $L^{\prime}=L_{C}+L_{D}$, where $L_{C}$ contains those triangles of $L^{\prime}$ in $C$. Say

$$
L_{C} \rightarrow K+K^{*} ; \text { then } L_{D} \rightarrow K^{*} \text {. }
$$

$K^{*}$ is a $\left(3 \epsilon_{1}, 1\right)$-cycle lying in $C \cdot D=\gamma$; it bounds an $(\epsilon, 2)$-chain $L^{*}$ in $\gamma$. Hence 


$$
L_{C}+L^{*} \rightarrow\left(K+K^{*}\right)+K^{*}=K .
$$

$L_{C}+L^{*}$ is an $(\epsilon, 2)$-chain in $C$, and the lemma is proved.

Lemma N. Let $A \cdot B=\gamma$, an arc whose end points are $a$ and $b$. Let the arcs $\alpha$ and $\beta$ join $a$ and $b$ in $A$ and $B$ respectively, neither having any points other than $a$ and $b$ in common with $\gamma$. If $\alpha+\beta \sim 0$ in $A+B$, then $\alpha+\gamma \sim 0$ in $A$.

Given an $\epsilon>0$, choose $\epsilon_{1}, \epsilon_{2}$ and $\delta$ as in the last lemma. Take $(\delta, 1)$-chains $K_{\alpha}, K_{\beta}$ and $K_{\gamma}$ in $\alpha, \beta$ and $\gamma$ respectively, each bounded by $a+b$; by Lemma J, it is sufficient to show that $K_{\alpha}+K_{\gamma} \epsilon \sim 0$ in $A$.

$K_{\alpha}+K_{\beta}$ bounds an $\left(\epsilon_{2}, 2\right)$-chain $L$ in $A+B$; we move each vertex of $L$ in $B \cdot V_{\epsilon_{2}}(A)-\gamma$ onto $\gamma$, giving a $\left(3 \epsilon_{1}, 2\right)$-chain $L^{\prime} \rightarrow K_{\alpha}+K_{\beta}^{\prime}$. Say $L^{\prime}=L_{A}$ $+L_{B}$, where $L_{A} \subset A, L_{B} \subset B$. If $L_{A} \rightarrow K_{\alpha}+K^{*}$, then $L_{B} \rightarrow K_{\beta}^{\prime}+K^{*}$, and $K^{*} \subset \gamma . K^{*}+K_{\gamma}$ is a $\left(3 \epsilon_{1}, 1\right)$-cycle on $\gamma$ bounding an $(\epsilon, 2)$-chain $L^{*}$ in $\gamma$. Hence $L_{A}+L^{*} \rightarrow K_{\alpha}+K_{\gamma}$ in $A$, completing the proof.

Lemma O. Let $\alpha, \beta$ and $\gamma$ be three arcs such that $\alpha \cdot \beta=\alpha \cdot \gamma=\beta \cdot \gamma=a+b$. Say $\alpha+\gamma \subset A$ and $\beta+\gamma \subset B$. If $\alpha+\gamma \sim 0$ in $A$ and $\beta+\gamma \sim 0$ in $B$, then $\alpha+\beta \sim 0$ in $A+B$.

Define $K_{\alpha}, K_{\beta}, K_{\gamma}$ as before; we need merely show that $K_{\alpha}+K_{\beta} \epsilon \sim 0$ in $A+B$. There are $(\epsilon, 2)$-chains $L_{A}$ and $L_{B}$ such that $L_{A} \rightarrow K_{\alpha}+K_{\gamma}$ in $A$ and $L_{B} \rightarrow K_{\beta}+K_{\gamma}$ in $B$; hence $L_{A}+L_{B} \rightarrow K_{\alpha}+K_{\beta}$ in $A+B$.

8. The set $R-\gamma$. Let $\gamma$ be any arc in $R$ crossing $J$; say the end points of $\gamma$ divide $J$ into the two $\operatorname{arcs} \alpha$ and $\beta$. By condition (2) of the theorem, $R-\gamma$ is not connected. Let $A^{\prime}$ and $B^{\prime}$ be those components of $R-\gamma$ containing $\langle\alpha\rangle \dagger$ and $\langle\beta\rangle$ respectively. These are not the same component. For if they were, putting $A=A^{\prime}+\gamma, D=R-A^{\prime}$, we have $J \subset A, J \sim 0$ in $R=A+D$, and $A \cdot D=\gamma$; hence, by Lemma $\mathrm{M}, J \sim 0$ in $A$, a proper subset of $R$, contrary to condition (1) of the theorem.

The same reasoning shows that $R$ has no cut point $p$; we need merely replace $\gamma$ by $p$ in Lemma $M$ and above.

Put

$$
A=A^{\prime}+\gamma, B=B^{\prime}+\gamma .
$$

If $D=R-A^{\prime}$, then $A \cdot D=\gamma$ and $J=\alpha+\beta \sim 0$ in $R=A+D$. Hence, by Lemma $\mathrm{N}, \alpha+\gamma \sim 0$ in $A$. Similarly, $\beta+\gamma \sim 0$ in $B$. Consequently, by Lemma $\mathrm{O}$, $J \sim 0$ in $A+B$, from which follows that $A+B=R$.

Moreover, $\alpha+\gamma$ is irreducibly $\sim 0$ in $A$. For if $\alpha+\gamma \sim 0$ in $A^{*}, \alpha+\gamma \subset A^{*}$ c $A$, then, by Lemma $\mathrm{O}, \alpha+\beta \sim 0$ in $A^{*}+B$; hence $A^{*}+B=R$, which is only possible if $A^{*}=A$. Similarly, $\beta+\gamma$ is irreducibly $\sim 0$ in $B$.

$\dagger\langle\alpha\rangle$ is $\alpha$ except for its end points, etc. 
Let us show that $A$ is a continuous curve. It is connected, as $A^{\prime}$ is; it is self-compact, being a closed subset of a compact space. $A$ is locally connected. For if $p$ and $q$ are points of $A$ close enough together, there is an arc $p q$ in $R$ of small diameter; if $p q$ lies partly in $B^{\prime}$, we can replace that part of it by an arc of $\gamma$ of small diameter. Lemma A now applies. Similarly, $B$ is a continuous curve.

9. We shall now show that any arc $\delta$ crossing $J_{A}=\alpha+\gamma$ in $A$ divides $A$. The following two lemmas will be useful.

LEMMA P. If $\eta_{1}$ and $\eta_{2}$ are arcs contained within the arcs $\gamma$ and $\beta$ respectively, then there is an arc $p q$ crossing $J_{B}=\beta+\gamma$ in $B$, with $p \subset \eta_{1}, q \subset \eta_{2}$.

This is an immediate consequence of Lemma $L$, if we take, for the closed sets of that lemma, the closed intervals of $J_{B}$ complementary to $\eta_{1}$ and $\eta_{2}$.

LEMMA Q. There are no two arcs $a b$ and $c d$ in $R$ without common points, each crossing $J$, whose end points are in the order acbd on $J$.

This follows directly from what we have seen above.

To show that $\delta$ divides $A$, we must consider four cases.

Case 1. Both end points of $\delta$ lie on $\alpha$. Suppose $A-\delta$ is connected; then it is arcwise connected, by Lemma $\mathrm{E}$. Hence there is an arc in $A-\delta$ joining a point $p$ of $\alpha$ lying between the two end points of $\delta$ and a point $q$ within $\gamma$. If $\eta_{1}$ is an arc within $\gamma$ containing $q$, there is an arc $r s$ in $B$ joining $\eta_{1}$ to a point $s$ within $\beta$, with only its end points $r$ and $s$ on $J_{B}$, by Lemma $\mathrm{P}$. The arc pqrs crosses $J$ and does not touch $\delta$. But the end points of this arc alternate with those of $\delta$ on $J$, contradicting Lemma Q.

Case 2. $\delta$ is an $\operatorname{arc} c d$, where $c$ lies within $\alpha, d$ lies within $\gamma$. If $A-\delta$ is connected, let $p q$ be an arc in this set joining points of $\alpha$ on opposite sides of $c$. If $\eta_{1}$ is an arc of $\gamma$ containing $d$ but not touching $p q$, let the arc $r s$ join $\eta_{1}$ to $\beta$ in $B$; then the arcs $p q$ and $c d r s$ contradict Lemma $Q$.

Case 3. The end points $c$ and $d$ of $\delta$ lie within $\gamma=a b$, say in the order $a c d b$. If $A-\delta$ is connected, let $p q$ be an arc in this set joining a point $p$ within $\alpha$ to a point $q$ in $\gamma$ between $c$ and $d$. If $\eta_{1}$ is an arc of $\gamma$ containing $q$ but not touching $\delta$, let $r_{1} s_{1}$ be an arc in $B$ joining $\eta_{1}$ to a point $s_{1}$ within $\beta$.

The arcs $a c r_{1}$ of $\gamma$ and $r_{1} s_{1}$ form an arc $a c r_{1} s_{1}$ crossing $J$; hence

$$
R-\operatorname{acr}_{1} s_{1}=C_{1}+C_{2},
$$

where $C_{1}$ contains the open arc $\left\langle a s_{1}>\right.$ of $\beta$, and $C_{2}$ contains $b$ and points connected with $b$. As $r_{1} s_{1}$ lies in $B, A^{\prime} \subset C_{1}+C_{2}$; the connected set $A^{\prime}+b$ lies thus in $C_{2}$. If $\eta_{2}$ is an arc of $\gamma$ containing $c$ but not touching $\eta_{1}$, and $r_{2} s_{2}$ is an arc in $C_{1}$ joining $\eta_{2}$ to a point $s_{2}$ of $\beta$ between $a$ and $s_{1}$, then $\eta_{2}+r_{2} s_{2}$ does not touch $p q r_{1} s_{1}$, and has only the point $c$ in common with $\delta$. 
Similarly, if $\eta_{3}$ is an arc of $\gamma$ containing $d$ but not touching $\eta_{1}$, there is an $\operatorname{arc} r_{3} s_{3}$ in $R$ - $-b d r_{1} s_{1}$ such that $r_{3}$ lies in $\eta_{3}, s_{3}$ lies in $\beta$ between $s_{1}$ and $b$, and $\eta_{3}+r_{3} s_{3}$ does not touch $p q r_{1} s_{1}$ and has only the point $d$ in common with $\delta$. The arc $r_{3} s_{3}$ does not touch $r_{2} s_{2}$, as it lies in $C_{2}$. Thus the two arcs $p q r_{1} s_{1}$ and $s_{2} r_{2} c d r_{3} s_{3}(c d=\delta)$ contradict Lemma Q.

Case 4. The same as Case 3, except that $c=a$ or $d=b$, say the latter. Then, in the notation of Case 3, the arcs $p q r_{1} s_{1}$ and $s_{2} r_{2} c b(c b=\delta)$ contradict Lemma $Q$.

This completes the proof that $A$ and $J_{A}\left(B\right.$ and $\left.J_{B}\right)$ satisfy the conditions of Theorem I.

10. The cutting up of $R$. We are concerned with the following lemma.

LEMMA R. $R$ may be cut into a finite number of pieces of arbitrarily small diameter.

Given an $\epsilon>0$, choose $\delta<\epsilon$ so as to satisfy the requirement in Lemma F. Suppose $R$ is cut up so that the diameter of the boundary of each piece is $<\delta$. Then each piece is of diameter $<3 \epsilon$. For otherwise there is a point $q$ of some piece $R_{i}$ at a distance $\geqq \epsilon$ from its boundary $J_{i}$. Let $p$ be a point of $J_{i}$, and $q^{\prime}$, a point of $R-R_{i}$ at a distance $\geqq \epsilon$ from $p$. Every arc from $q$ to $q^{\prime}$ must cut the boundary $J_{i}$ of $R_{i}$ and thus must pass within $\delta$ of $p$, contradicting Lemma $F$.

The lemma thus follows from

Lemma S. Given a $\delta>0, R$ can be cut up so that the diameter of the boundary of each piece is $<\delta$.

Express $R$ as the union of a finite number of continua:

$$
R=K_{1}+K_{2}+\cdots+K_{m}, \delta\left(K_{i}\right)<\delta / 2 .
$$

We shall cut up $R$ in such a manner that no two of these continua $K_{i}$ and $K_{j}$ have points on the boundary of the same piece of $R$, if $K_{i} \cdot K_{j}=0$; the lemma will then follow.

Suppose we have cut $R$ up a certain amount (perhaps not yet at all), into the pieces $R_{1}, R_{2}, \cdots, R_{n}$, with boundaries $J_{1}, J_{2}, \cdots, J_{n}$ (we may have $R$ and $J$ alone). Of course each boundary $J_{i}$ separates $R_{i}$ from the rest of $R$. Take any two continua, say $K_{1}$ and $K_{2}$, with $K_{1} \cdot K_{2}=0$, each of which has points on one of these $J_{i}$, say $J_{1}$. We shall cut $R$ up further so that in the new pieces there is no one (i.e. no piece, not merely no boundary of a piece) which has any points in common with both $K_{1}$ and $K_{2}$; then on any further cutting up of $R$, this will still be true.

Divide the points of $J_{1}$ into three sets, as follows. We put a point $x$ into the first set if it lies in $K_{1}$, or if following $J_{1}$ in both directions we reach points 
of $K_{1}$ before reaching points of $K_{2}$; we put $x$ into the second set if the same conditions hold with $K_{1}$ and $K_{2}$ interchanged; all other points we put into the third set. This set $L_{3}^{\prime}$ consists of open intervals of $J_{1}$, each being bounded by a point of $K_{1}$ on one end and a point of $K_{2}$ on the other. The points of the first set together with the points $K_{1} \cdot R_{1}$ form a closed set $L_{1}$, and those of the second set together with $K_{2} \cdot R_{1}$ form a closed set $L_{2}$. Then $\rho\left(L_{1}, L_{2}\right)>0$ as $L_{1} \cdot L_{2}=0$, from which follows that there are but a finite number of intervals in $L_{3}^{\prime}$. As $K_{1}$ is connected, each component of $L_{1}$ has points on $J_{1}$, and thus on one of the intervals $L_{3}$ of $J_{1}$ complementary to the intervals of $L_{3}^{\prime}$. Thus there are a finite number of components $L_{11}, L_{12}, \cdots, L_{1 m_{1}}$ in $L_{1}$. Similarly there are a finite number of components $L_{21}, L_{22}, \cdots, L_{2 m_{2}}$ in $L_{2}$.

We shall now cut $R_{1}$ into a number of pieces, in each of which either $K_{1}$ has no points or $K_{2}$ has no points. Suppose $L_{31}{ }^{\prime}, \cdots, L_{3 m_{3}}{ }^{\prime}$ and $L_{31}, \cdots, L_{3 m_{3}}$ are the intervals of $L_{3}^{\prime}$ and $L_{3}$ respectively, and say they lie in the order $L_{31}$, $L_{31}^{\prime}, L_{32}, L_{32}^{\prime}, \cdots, L_{3 m_{3}}, L_{3 m_{3}^{\prime}}^{\prime}$ on $J_{1}$. If we go around $J_{1}$, the intervals of $L_{3}$ lie alternately in $L_{1}$ and $L_{2}$. Starting at $L_{31}$, which lies in $L_{11}$ say, go around $J_{1}$ till we reach another interval $L_{3 k}$ in $L_{11}$ (we may have gotten back to $L_{31}$ ). Put $L_{32}, L_{3, k-1}$ and all of $J_{1}$ between these into a set $M_{2}^{\prime}$ (which may be $L_{32}$ alone), and put $L_{3 k}, L_{31}$, and all of $J_{1}$ between these on the other side from $L_{32}$ into a set $M_{1}^{\prime}$ (which may be $L_{31}$ alone). $L_{31}^{\prime}$ and $L_{3, k-1}^{\prime}$ are the two intervals of $J_{1}$ complementary to $M_{1}^{\prime}$ and $M_{2}^{\prime}$.

No set $L_{1 i}$ or $L_{2 j}$ has points in both $M_{1}^{\prime}$ and $M_{2}^{\prime}$. This follows for $L_{11}$ by construction. If it were false for some other set, say $L_{18}$, then $L_{18}$ would have points on two intervals $L_{3 p}$ and $L_{3 q}$ separated by $L_{31}$ and $L_{3 k}$ on $J_{1}$. Now $L_{11} \cdot L_{18}=0$, hence $\rho\left(L_{11}, L_{18}\right)>0$. As $R_{1}$ is a continuous curve, there are continuous curves $L_{11}{ }^{*}$ and $L_{18}{ }^{*}$ in $R_{1}$ containing $L_{11}$ and $L_{18}$ and such that $L_{11}{ }^{*} \cdot L_{18}{ }^{*}=0$ (see Lemma $\mathrm{C}$ ). These sets are arcwise connected, and we can draw arcs contradicting Lemma $\mathrm{Q}$.

Let $M_{1}$ be $M_{1}^{\prime}$ plus all components $L_{1 i}$ and $L_{2 j}$ containing points of $M_{1}^{\prime}$, and define $M_{2}$ similarly. Then $M_{1}$ and $M_{2}$ are closed, $M_{1} \cdot M_{2}=0$, and $M_{1}+M_{2}$ $>L_{1}+L_{2}$. By Lemma $L$ we can draw an $\operatorname{arc} \gamma_{1}$ from $L_{31}^{\prime}$ to $L_{3, k-1}^{\prime}$ which has no points in $M_{1}$ or in $M_{2} . R_{1}$ is thus cut into two pieces, in each of which there is at least one component $L_{1 i}$ or $L_{2 j}$; for one contains $L_{11}$, and the other contains that $L_{2 j}$ containing $L_{32}$. Thus in each piece there are less than $m_{1}+m_{2}$ components, the number in $R_{1}$.

If one of the resulting pieces contains more than one component, we cut it up, etc. Finally each new piece of $R_{1}$ has points of only one component, and thus $K_{1}$ and $K_{2}$ are separated in $R_{1}$. We now separate $K_{1}$ and $K_{2}$ in each other piece $R_{i}$ of $R$ also. This is possible, for if $K_{i}(i=1,2)$ has points in any $R_{k}$, it also has points on $J_{k}$. 
If now there are any other two of the continua $K_{i}$ and $K_{j}, K_{i} \cdot K_{j}=0$, each of which has points on some new $J_{k}$, we cut $R$ further till this is no longer true, etc. This completes the proof.

11. The homeomorphism. Cut $R$ into pieces of diameter < some $\sigma$. We make corresponding cuts in $R^{\prime}$ as follows. The first arc $\gamma$ drawn in $R$ cuts $R$ into the two pieces $R_{1}$ and $R_{2}$ with boundaries $J_{1}$ and $J_{2}$ say. Draw any arc $\gamma^{\prime}$ crossing $J^{\prime}$ in $R^{\prime}$, cutting $R^{\prime}$ into the pieces $R_{1}^{\prime}$ and $R_{2}^{\prime}$ with boundaries $J_{1}^{\prime}$ and $J_{2}^{\prime}$. We note that $J_{1}^{\prime}+J_{2}^{\prime}$ is homeomorphic with $J_{1}+J_{2}$, with $J_{k}^{\prime}$ corresponding to $J_{k}, k=1,2$. Say $\gamma_{1}$ is an arc in $R_{1}$, cutting $R_{1}$ into pieces $R_{11}$ and $R_{12}$ with boundaries $J_{11}$ and $J_{12}$. If $a_{1}$ and $b_{1}$ are the end points of $\gamma_{1}$, let $a_{1}{ }^{*}$ and $a_{2}{ }^{*}$ be the corresponding points of $J_{1}^{\prime}$ in the above homeomorphism. Draw an arc $\gamma_{1}^{\prime}$ crossing $J_{1}^{\prime}$ in $R_{1}^{\prime}$, with end points $a_{1}^{\prime}$ and $b_{1}^{\prime}$ close to $a_{1}{ }^{*}$ and $b_{1}{ }^{*}$ respectively (Lemma P); $R_{1}^{\prime}$ is divided thereby into the pieces $R_{11}{ }^{\prime}$ and $R_{12}^{\prime}$ with boundaries $J_{11}^{\prime}$ and $J_{12}^{\prime}$. Moreover, $J_{11}^{\prime}+J_{12}^{\prime}+J_{2}^{\prime}$ is homeomorphic with $J_{11}+J_{12}+J_{2}$, with boundaries with the same subscripts corresponding.

In general, suppose $R_{i_{1} i_{2} \cdots i_{m}}$ is a piece that is present after $R$ is cut a certain amount, and say the $\operatorname{arc} \gamma_{i_{1}} \ldots i_{m}$ divides this set into the pieces $R_{i_{1}} \cdots i_{i^{1}}$ and $R_{i_{1}} \ldots i_{i_{m}}$, with boundaries $J_{i_{1}} \cdots i_{i^{1}}$ and $J_{i_{1}} \ldots i_{m^{2}}$. If $a_{i_{1}} \cdots i_{m}$ and $b_{i_{1}} \ldots i_{m}$ are the end points of $\gamma_{i_{1}} \ldots i_{m}$, let $a_{i_{1}}{ }^{*} \ldots i_{m}$ and $b_{i_{1}}{ }^{*} \ldots i_{m}$ be the corresponding points on $J !_{1}^{\prime} \ldots i_{m}$ in the homeomorphism we have already. Draw an arc $\boldsymbol{\gamma}_{i_{1}}^{\prime} \ldots i_{m}$ crossing $J_{i_{1}}^{\prime} \ldots i_{m}$, with end points $a_{i_{1}}{ }^{\prime} \ldots i_{m}$ and $b_{i_{1}}{ }^{\prime} \ldots i_{m}$ close to the above points, dividing $R_{i_{1}}{ }^{\prime}{ }^{\prime} i_{m}$ into the pieces $R_{i_{1}}{ }^{\prime} \ldots i_{i^{1}}$ and $R_{i_{1}}{ }^{\prime} \ldots i_{m^{2}}$, with boundaries $J_{i_{1}^{\prime}}^{\prime} \ldots i_{m}{ }^{1}$ and $J_{i_{1}^{\prime}}^{\prime} \ldots i_{m}^{2}$. The set of boundaries with primes is now homeomorphic with the set of boundaries without primes, boundaries with the same subscripts corresponding. We note that if $R_{i_{1}} \cdots_{i_{m}}$ and $R_{j_{1}} \cdots i_{m}$ have common points, then $R_{i_{1}}{ }^{\prime} \ldots i_{m}$ and $R_{j_{1}}{ }^{\prime} \ldots i_{m}$ have common points, and conversely.

Having cut $R$ into pieces of diameter $<\sigma$ and having cut $R^{\prime}$ in a corresponding fashion, we now cut each piece of $R^{\prime}$ into pieces of diameter $<\sigma / 2$ and cut each piece of $R$ in a corresponding fashion. Next we cut each resulting piece of $R$ into pieces of diameter $<\sigma / 4$, etc. Now for any $\epsilon>0$ there is an $m$ such that

$$
\delta\left(R_{i_{1}} \cdots i_{m}\right)<\epsilon, \quad \delta\left(R_{i_{1}}^{\prime} \cdots i_{m}\right)<\epsilon,
$$

for any $m$-fold subscript.

We now establish the homeomorphism between $R$ and $R^{\prime}$. Let $p$ be any point of $R$. It lies in either $R_{1}$ or $R_{2}$ (perhaps in both), say in $R_{i_{1}}$. Then it lies in either $R_{i_{1} 1}$ or $R_{i_{1} 2}$ (perhaps in both), say in $R_{i_{1} i_{2}}$, etc. Thus we have a sequence of pieces 


$$
R \supset R_{i_{1}} \supset R_{i_{1} i_{2}} \supset \cdots \supset p .
$$

The corresponding pieces in $R^{\prime}$ have a single limit point:

$$
R^{\prime} \supset R_{i_{1}}^{\prime} \supset R_{i_{1} i_{2}}^{\prime} \supset \cdots \supset p^{\prime} \text {. }
$$

This point $p^{\prime}$ we let correspond to $p$.

If there are different sequences of pieces in $R$ containing $p$, we have different sequences in $R^{\prime}$ defining points $p^{\prime}$. However, all these points $p^{\prime}$ are the same. For if $R, R_{i_{1}}, R_{i_{1} i_{2}}, \cdots$, and $R, R_{j_{1}}, R_{j_{1} j_{2}}, \cdots$, are two sequences containing $p$, then each piece $R_{i_{1} \ldots i_{m}}$ has points in common with $R_{j_{1}} \ldots j_{m}$, namely, the point $p$; hence, as we saw above, $R_{i_{1}}{ }^{\prime} \ldots i_{m}$ and $R_{j_{1}}{ }^{\prime} \ldots i_{m}$ have common points. Thus the corresponding sequences in $R^{\prime}$ close down on a single point. Similarly, to each point $p^{\prime}$ in $R^{\prime}$ corresponds a single point $p$ in $R$.

Finally, the correspondence is continuous. For take a point $p$ in $R$ and an $\epsilon>0$. Let $p^{\prime}$ be the corresponding point in $R^{\prime}$, and choose an $m$ so that $\delta\left(R_{i_{1}}{ }^{\prime} \ldots i_{m}\right)<\epsilon$ for all $m$-fold subscripts. Consider all the $R_{i_{1}} \ldots i_{m}$ with $m$-fold subscripts which contain $p$; these include all points of $R$ in some $V_{\delta}(p)$. Then if $q \subset V_{\delta}(p)$, the corresponding point $q^{\prime}$ is in $V_{\epsilon}\left(p^{\prime}\right)$, and the continuity is established. This completes the proof of Theorem $\mathrm{I}$.

12. Proof of Theorem II. Let $I$ be a circle in the plane, and let $S$ be $I$ plus its interior. $S$ is self-compact, connected and locally connected, and is thus a continuous curve. That $I \sim 0$ in $S$ follows from Lemma K. $\dagger$

To show that $I$ is irreducibly $\sim 0$ in $S$, suppose that $I \sim 0$ in $S^{\prime}$, a proper closed subset of $S$; we can suppose that $S^{\prime}$ is a continuous curve. Let $p$ be a point of $S$ not in $S^{\prime}$, and let $V_{\delta}(p)$ have no points in $S^{\prime}$. Let $a b$ be a segment of a straight line passing through $p$ with its ends on $I$. Let $a_{1} b_{1}$ and $a_{2} b_{2}$ be parallel segments enclosing $a b$, and lying at a distance $\delta$ from $a b$. Then in that portion of $S^{\prime}$ between $a_{1} b_{1}$ and $a_{2} b_{2}$, the (short) arcs $a_{1} a_{2}$ and $b_{1} b_{2}$ are not connected. But if $C$ and $D$ are those parts of $S^{\prime}$ outside $a_{1} b_{1}$ and $a_{2} b_{2}$, by Lemma $\mathrm{L}$ we can draw an arc joining $a_{1} a_{2}$ to $b_{1} b_{2}$ in $S^{\prime}-(C+D)$, a contradiction.

Finally, that an arc crossing $I$ in $S$ divides $S$ is a special (and easily proved) case of the Jordan theorem. This completes the proof.

13. The Jordan theorem. Let $J$ be a simple closed curve in the plane. Let $I$ be a circle containing $J$ in its interior. Draw two non-intersecting line segments from $I$ to $J . S=I$ plus its interior is thus cut into three closed 2-cells, one of which, say $R$, has the boundary $J$. Then $R-J$ is the inside of $J$. The points of $J$ are obviously accessible from either side.

$\dagger$ For $S$ is a closed 2-cell.

Princeton University, Princeton, N. J. 